\title{
Simplex Optimization and Its Applicability for Solving Analytical Problems
}

\author{
Anna Maria Michałowska-Kaczmarczyk1, Tadeusz Michałowski2* \\ ${ }^{1}$ Department of Oncology, The University Hospital in Cracow, Cracow, Poland \\ ${ }^{2}$ Faculty of Engineering and Chemical Technology, Cracow University of Technology, Cracow, Poland \\ Email: "michalot@o2.pl
}

Received 14 April 2014; revised 14 May 2014; accepted 21 May 2014

Copyright (C) 2014 by authors and Scientific Research Publishing Inc.

This work is licensed under the Creative Commons Attribution International License (CC BY).

http://creativecommons.org/licenses/by/4.0/

(c) (i) Open Access

\begin{abstract}
Formulation of the simplex matrix referred to $n-D$ space, is presented in terms of the scalar product of vectors, known from elementary algebra. The principles of a simplex optimization procedure are presented on a simple example, with use of a target function taken as a criterion of optimization, where accuracy and precision are treated equally in searching optimal conditions of a gravimetric analysis.
\end{abstract}

Keywords

Simplex, Simplex Optimization Procedure

\section{Introduction}

Simplex is a geometric figure, formed on the basis of $n+1$ points $A_{i}(i=0, \cdots, n)$ in the $n$-dimensional space, i.e., a number of the points exceeds the dimension of the space by one. These points are referred to as the vertices of the simplex. Distribution of the points $A_{i}$ in this space is represented by a matrix of this simplex. Specifying the coordinates for $A_{i}$ is easy if it concerns the 2-D or 3-D space, but it becomes less comprehensible when the issue concerns the $n$-dimensional $(n-D)$ simplex. Although in the literature [1] one can find ready-made forms of the simplex matrix in $n-D$ space, but information on how to receive it in an easy manner is lacking.

The simplex concept is the basis for the Nelder-Mead [2] simplex optimization procedure (SOP) [3], conceived as a modification of the simplex method of Spendley, Hext and Himsworth [4]. The SOP is easy to use, and does not require calculation of derivatives [5] [6], as in the gradient (local steepest descent) method [7] [8]). The SOP

\footnotetext{
*Corresponding author.
}

How to cite this paper: Michałowska-Kaczmarczyk, A.M. and Michałowski, T. (2014) Simplex Optimization and Its Applicability for Solving Analytical Problems. Journal of Applied Mathematics and Physics, 2, 723-736.

http://dx.doi.org/10.4236/jamp.2014.27080 
is widely applicable and popular in different fields of chemistry, chemical engineering, and medicine [9]. Furthermore, it works well in practice on a wide variety of problems, where real-valued minimization functions with scalar variables are applied.

The sequential simplex method is considered as one of the most effective and robust methods applied in methodology of Evolutionary Operation (EVOP) experimental design [10]-[15]. The SOP method was implemented in MINUIT (a Fortran from CERN [16] package), then in Pascal, Modula-2, Visual Basic, and C++ [17]. The iterations are stopped when the function minimized is less than a preset value.

In this article, the problem of obtaining the simplex matrix in $n-D$ space will be presented on the basis of the triangle and the scalar product concept, well-known to the students from earlier stages of education. The evolution of the simplex according to SOP [3] [18] will also be presented in a understandable manner.

\section{Simplexes in 2-D and 3-D Space}

In order to introduce the simplex concept, we start from the point $(0-D)$, (I) segment (1-D), (II) equilateral triangle $(2-D)$, and (III) tetrahedron $(3-D)$ concepts, known from the elementary geometry. The $0-D, 1-D$, $2-D, 3-D$ (and generally $n-D$ ) notations express the dimensionality of the corresponding geometrical entities (Figure 1).

The points: $A_{0}, A_{1}, A_{2}$ are vertices of the triangle $A_{0} A_{1} A_{2}$; the points: $A_{0}, A_{1}, A_{2}, A_{3}$ are the vertices of the tetrahedron $A_{0} A_{1} A_{2} A_{3} . A_{0} A_{1}$ is one of the edges of the triangle; $A_{0} A_{1}$ is one of the edges of the tetrahedron. Equilateral triangle $A_{0} A_{1} A_{2}$ is one of walls of the tetrahedron.

Segment, equilateral triangle, and tetrahedron can be presented in the same scale; it means that all distances, equal to unity (chosen arbitrarily), between the (neighbouring) points $A_{i}$ and $A_{j}$ of a polygon are assumed, i.e. the length $\left|A_{i} A_{j}\right|=1$.

All angles between edges of the equilateral triangle are equal to $60^{\circ}(\pi / 3 \mathrm{rd})$. All angles between adjacent tetrahedron edges are equal $60^{\circ}$ too, owing to the fact that all the tetrahedron walls are composed of equilateral triangles $(2-D)$. By turns, tetrahedrons form the $3-D$ walls in $4-D$ polygon, etc. It means that all angles between adjacent edges of a symmetrical $n-D$ polygon are equal $60^{\circ}$, as well.

The equilateral triangle can assume different positions on the plane. A particular case is the triangle with unit edges, "anchored" at the origin of co-ordinate axis, as one presented in Figure 2.

The successive vertices of the equilateral triangle can be presented in matrix form, where the rows are expressed by co-ordinates of the corresponding points $A_{i}(i=0,1,2)$ :

$$
\boldsymbol{X}=\left[\begin{array}{cc}
0 & 0 \\
1 & 0 \\
1 / 2 & \sqrt{3} / 2
\end{array}\right] \begin{aligned}
& A_{0} \\
& A_{1} \\
& A_{2}
\end{aligned}
$$

The second and third rows of this matrix are identical with components of transposed vectors: $A_{0} A_{1}$ and $A_{0} A_{2}$, respectively:

$$
A_{0} A_{1}=[1,0]^{\mathrm{T}}, \quad A_{0} A_{2}=[1 / 2,2]^{\mathrm{T}}
$$

The tetrahedron can assume different positions in $3-D$ space. A particular case is the tetrahedron anchored at the origin of co-ordinate axis as one presented in Figure 3.

The successive vertices of the tetrahedron can be presented in the matrix form:

$$
\boldsymbol{X}=\left[\begin{array}{ccc}
0 & 0 & 0 \\
1 & 0 & 0 \\
1 / 2 \sqrt{3} / 2 & 0 \\
1 / 2 & \sqrt{3} / 6 & \sqrt{6} / 3
\end{array}\right] \begin{aligned}
& A_{0} \\
& A_{1} \\
& A_{2} \\
& A_{3}
\end{aligned}
$$

The second, third and fourth rows of this matrix are identical with components of the transposed vectors: $A_{0} A_{1}, A_{0} A_{2}$ and $A_{0} A_{3}$, respectively:

$$
A_{0} A_{1}=[1,0,0]^{\mathrm{T}} ; A_{0} A_{2}=[1 / 2, \sqrt{3} / 2,0]^{\mathrm{T}} ; A_{0} A_{3}=[1 / 2, \sqrt{3} / 6, \sqrt{6} / 3]^{\mathrm{T}}
$$



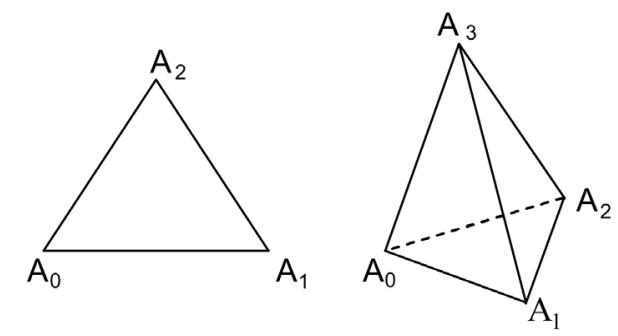

Figure 1. Equilateral triangle and tetrahedron.

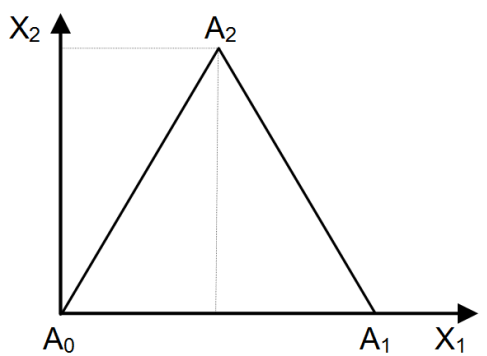

Figure 2. The equilateral triangle with unit edges and points: $A_{0}(0,0) ; A_{1}(1,0) ; A_{2}(0.5, \sqrt{3} / 2)$.

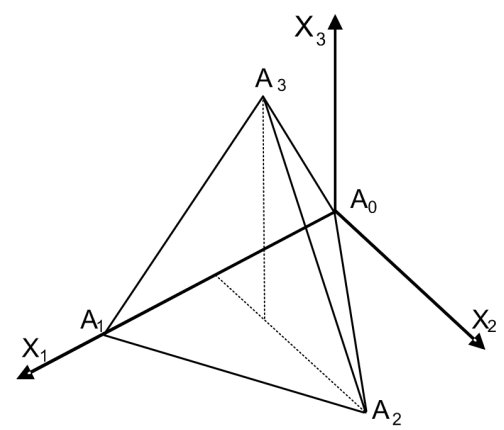

Figure 3. The tetrahedron with unit edges and $A_{0}(0,0,0) ; A_{1}(1,0,0) ; A_{2}(1 / 2, \sqrt{3} / 2,0)$;

$A_{3}(1 / 2, \sqrt{3} / 6, \sqrt{6} / 3)$.

\section{Simplexes in $n-D$ Space}

The segment, equilateral triangle and tetrahedron are imaginable concepts. However, application of the concepts involved with geometric figures of higher dimension $(n \geq 4)$ is beyond imagination. One should be taken into account that, in the simplex optimization, we are forced, as a rule, to consider higher number of factors influencing the optimizing process.

Although the figures in $n-D$ space (Cartesian co-ordinate system) are beyond imagination, one can present them in projective space. In Figure 4, any pair of points of the simplex is connected, informally, by a segment (line); the segments (sides, edges) are marked there with broken lines. Particularly, two points form the 1-D simplex in 1-D space. Adding a third point (that not lies along the segment or its extension) gives a triangle (2-D simplex) in 2-D space. Adding a fourth point in 3-D space (not coplanar with 2-D simplex plane), one obtains a tetrahedron (3-D simplex), etc. Generally, adding a successive $k+1$-th $(k=2,3, \cdots, n-1)$ point, do not lying in surface formed by $k-1-D$ simplex, one obtains the $k-D$ simplex. In other words, adding the successive point that not lies on (or in plane of) simplexes of lower dimension and connecting it, every time, with the remaining points, one can imagine the simplexes with larger and larger dimensions, in projective space. 


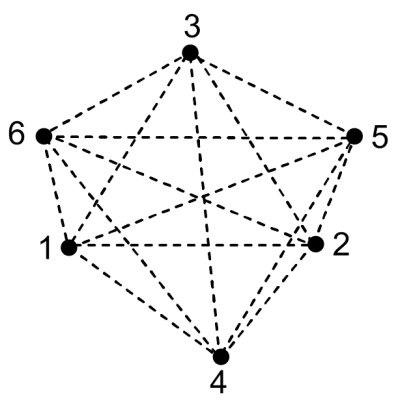

Figure 4. Formation of consecutive dimensions (up to 5- $D$ ) of a simplex in projective space.

Let us assume that the polygon is "anchored" in $n$ - $D$-space in such a manner that $A_{0}$ is in the origin of the co-ordinate axis and any successive point "enters" the new dimension of the $n$ - $D$-space, see Figure 4 . It means that the co-ordinates of the corresponding points of $n-D$ polygon are as follows:

$$
\begin{aligned}
& A_{0}=(0,0,0, \cdots, 0,0) ; A_{1}=\left(x_{11}, 0,0, \cdots, 0,0\right) ; A_{2}=\left(x_{21}, x_{22}, 0, \cdots, 0,0\right) ; A_{3}=\left(x_{31}, x_{32}, x_{33}, \cdots, 0,0\right) ; \cdots ; \\
& A_{n-1}=\left(x_{n-1,1}, x_{n-1,2}, x_{n-1,3}, \cdots, x_{n-1, n-1}, 0\right) ; A_{n}=\left(x_{n 1}, x_{n 2}, x_{n 3}, \cdots, x_{n, n-1}, x_{n n}\right) .
\end{aligned}
$$

This means that $x_{i j}=0$ for $j>i$.

As stated above, the number of vertices exceeds, by 1 , the dimension of the space; it means that the polygon in $n-D$ space involves $n+1$ vertices.

We consider a pencil of $n$ unit vectors, $x_{i}=A_{0} A_{i}=\left[x_{i 1}, x_{i 2}, x_{i 3}, \cdots, x_{i i}, \cdots, x_{i n}\right]^{\mathrm{T}}$, all starting from the point $A_{0}=(0, \cdots, 0)$, i.e., origin of co-ordinates of $n-D$ space and ending at the points $A_{i}(i=1,2, \cdots, n)$. One should notice that the basic components $x_{i j}$ of the vector $\boldsymbol{x}_{i}$ starting at the origin of co-ordinates, are identical with co-ordinates of the point $A_{i}=\left(x_{i 1}, x_{i 2}, x_{i 3}, \cdots, x_{i j}, \cdots, x_{i n}\right)$. Then for the vectors $A_{0} A_{i}$ based on the points specified in (3) we have:

$$
\begin{aligned}
& \boldsymbol{x}_{1}=A_{0} A_{1}=\left[x_{11}, 0,0, \cdots, 0\right]^{\mathrm{T}} ; \\
& \boldsymbol{x}_{2}=A_{0} A_{2}=\left[x_{21}, x_{22}, 0, \cdots, 0\right]^{\mathrm{T}} ; \\
& \boldsymbol{x}_{3}=A_{0} A_{3}=\left[x_{31}, x_{32}, x_{33}, 0, \cdots, 0\right]^{\mathrm{T}} ; \\
& \boldsymbol{x}_{i}=A_{0} A_{i}=\left[x_{i 1}, x_{i 2}, x_{i 3}, \cdots, x_{i i}, 0, \cdots, 0\right]^{\mathrm{T}} ; \\
& \boldsymbol{x}_{n}=A_{0} A_{n}=\left[x_{n 1}, x_{n 2}, x_{n 3}, \cdots, x_{n i}, \cdots, x_{n n}\right]^{\mathrm{T}} .
\end{aligned}
$$

Let us construct the matrix $\boldsymbol{X}$ (Equation (5)) composed of the co-ordinates of the corresponding points in $n-D$ space:

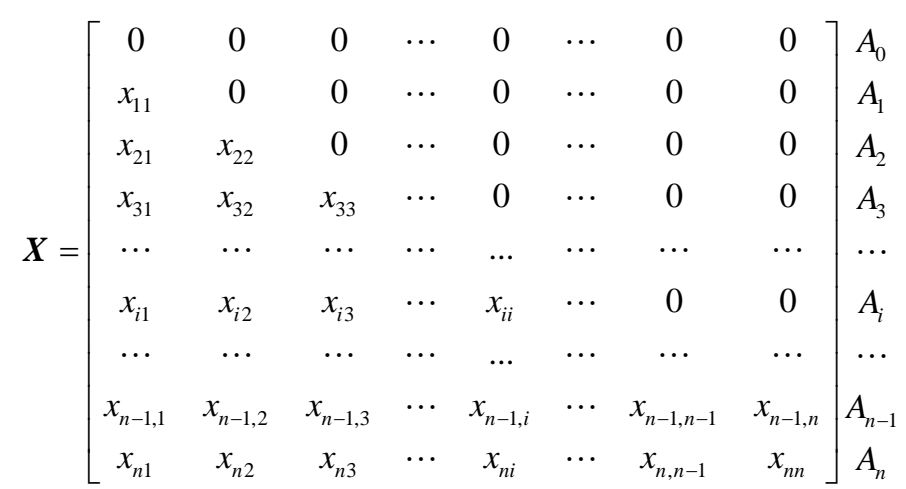

Moreover, we assume non-negative values for the coordinates, i.e. $x_{i j} \geq 0$, and then the elements of the ma- 
trix $\boldsymbol{X}=\left[x_{i j}\right]$ can be specified as follows:

$$
x_{i j}= \begin{cases}>0 & \text { for } i \geq j \\ 0 & \text { for } i<j \quad(i, j \in<1, n>) \\ 0 & \text { for } i=0 \quad(j=1, \cdots, n)\end{cases}
$$

Let $\boldsymbol{e}_{\boldsymbol{i}}$ be the unit vector along the $i$-th axis, $X_{i}$, i.e. $\left|\boldsymbol{e}_{i}\right|=1$. The $\boldsymbol{e}_{j}$ vectors form a set of mutually orthogonal vectors in $\mathrm{n}-D$ space. The scalar products:

$$
\boldsymbol{e}_{i} \cdot \boldsymbol{e}_{j}=\left|\boldsymbol{e}_{i}\right| \cdot\left|\boldsymbol{e}_{j}\right| \cdot \cos \left(\angle \boldsymbol{e}_{i}, \boldsymbol{e}_{j}\right)=\delta_{i j}=\left\{\begin{array}{lll}
1 & \text { for } & j=i \\
0 & \text { for } & j \neq i
\end{array}\right.
$$

where $\delta_{i j}$ is the Kronecker symbol.

Any vector $\boldsymbol{x}_{i}$ in $n-D$ space can be also written as $\boldsymbol{x}_{i}=\left[\mathrm{x}_{i 1}, \mathrm{x}_{i 2}, \cdots, \mathrm{x}_{i n}\right]^{\mathrm{T}} ; \boldsymbol{x}_{i}=\sum_{k=1}^{n} \boldsymbol{e}_{\mathrm{k}} \cdot x_{i k}$. Then the scalar product of two vectors, $\boldsymbol{x}_{i}$ and $\boldsymbol{x}_{j}$, in $n-D$ space is as follows:

$$
\boldsymbol{x}_{i} \cdot \boldsymbol{x}_{j}=\left|\boldsymbol{x}_{i}\right| \cdot\left|\boldsymbol{x}_{j}\right| \cdot \cos \left(\angle \boldsymbol{x}_{i}, \boldsymbol{x}_{j}\right)=\sum_{k=1}^{n} x_{i k} x_{j k}
$$

where $\angle \boldsymbol{x}_{i}, \boldsymbol{x}_{j}$ is the angle between vectors $\boldsymbol{x}_{i}$ and $\boldsymbol{x}_{j}$ spanning the simplex in $n-D$ space. The angle between different vectors $\boldsymbol{x}_{i}=A_{0} A_{i}$ and $\boldsymbol{x}_{j}=A_{0} A_{j}$ of $n-D$ simplex equals $\pi / 3$ [rd], i.e. $\angle \boldsymbol{x}_{i}, \boldsymbol{x}_{j}=60^{\circ}$ and then $\cos \left(\angle \boldsymbol{x}_{i}, \boldsymbol{x}_{j}\right)=\cos 60^{\circ}=1 / 2$, for any pair of the vectors $\boldsymbol{x}_{i}$ and $\boldsymbol{x}_{j}$ : in triangle $(2-D)$, in tetrahedron (3-D), . . Moreover, $\angle \boldsymbol{x}_{i}, \boldsymbol{x}_{i}=0$ and then $\cos \left(\angle \boldsymbol{x}_{i}, \boldsymbol{x}_{j}\right)=\cos 0^{\circ}=1$. If the unit vectors $\boldsymbol{x}_{i}(\mathrm{i}=1, \cdots, n)$ are assumed, i.e., $\left|\boldsymbol{x}_{i}\right|=x_{i}=1$, then from (8) and (4)-(6) we have:

$$
\begin{gathered}
\sum_{k=1}^{n} x_{i k} x_{j k}=\sum_{k=1}^{m} x_{i k} x_{j k}=\frac{1}{2} \text { where } m=\min (i, j) \\
\sum_{j=1}^{i} x_{i j}^{2}=1, \text { where } i=1, \cdots, n
\end{gathered}
$$

From (9), (10) and (6) we get, by turns,

$$
\begin{gathered}
x_{11}^{2}=1 \rightarrow x_{11}=1=\left(\frac{1+1}{2 \cdot 1}\right)^{1 / 2}=h_{1} \\
x_{11} \cdot x_{i 1}+0 \cdot x_{i 2}+\cdots+0 \cdot x_{i i}+0 \cdot 0+\cdots+0 \cdot 0=\frac{1}{2}, \text { i.e. } \\
x_{11} \cdot x_{i 1}=1 / 2(\text { for } i=2, \cdots, n) \rightarrow x_{i 1}=\frac{1}{2}=\frac{1}{1+1} \cdot h_{1} \quad(\text { for } 2 \leq i \leq n) \\
x_{21}^{2}+x_{22}^{2}=1 \rightarrow x_{22}=\left(1-x_{21}^{2}\right)^{1 / 2}=\left(1-\left(\frac{1}{2}\right)^{2}\right)^{1 / 2}=\frac{\sqrt{3}}{2}=\left(\frac{2+1}{2 \cdot 2}\right)^{1 / 2}=h_{2} \\
x_{21} \cdot x_{i 1}+x_{22} \cdot x_{i 2}=\frac{1}{2} ? \rightarrow x_{i 2}=\frac{1}{3} \frac{\sqrt{3}}{2}=\frac{1}{2+1} h_{2} \quad(\text { for } 3 \leq i \leq n) \\
x_{31}^{2}+x_{32}^{2}+x_{33}^{2}=1 \rightarrow x_{33}=\left(1-x_{31}^{2}-x_{32}^{2}\right)^{1 / 2}=\frac{\sqrt{6}}{3}=\left(\frac{3+1}{2 \cdot 3}\right)^{1 / 2}=h_{3} \\
x_{31} \cdot x_{i 1}+x_{32} \cdot x_{i 2}+x_{33} \cdot x_{i 3}=\frac{1}{2} \rightarrow x_{i 3}=\frac{1}{4} \frac{\sqrt{6}}{3}=\frac{1}{3+1} h_{3} \quad(\text { for } 4 \leq i \leq n) \\
x_{41}^{2}+x_{42}^{2}+x_{43}^{2}+x_{44}^{2}=1 \rightarrow x_{44}=\left(1-x_{41}^{2}-x_{42}^{2}-x_{43}^{2}\right)^{1 / 2}=\frac{\sqrt{10}}{4}=\left(\frac{4+1}{2 \cdot 4}\right)^{1 / 2}=h_{4}
\end{gathered}
$$




$$
x_{41} x_{i 1}+x_{42} x_{i 2}+x_{43} x_{i 3}+x_{44} x_{i 4}=\frac{1}{2} \rightarrow x_{i 4}=\frac{1}{5} \frac{\sqrt{10}}{4}=\frac{1}{4+1} h_{4} \quad(\text { for } 5 \leq i \leq n)
$$

and so forth. On this basis, one can write:

$$
\begin{gathered}
x_{j j}=h_{j}=\left(\frac{j+1}{2 j}\right)^{1 / 2} \quad(\text { for } 1 \leq j \leq n) \\
x_{i j}=r_{j}=\frac{1}{j+1}\left(\frac{j+1}{2 j}\right)^{1 / 2}=\left(\frac{1}{2 j(j+1)}\right)^{1 / 2} \quad(\text { for } j<i \leq n)
\end{gathered}
$$

The elements $x_{i j}$ of the matrix $\boldsymbol{X}=\left[x_{i j}\right]$ (Equation (5)) are thus derived with use of principles of elementary algebra and geometry. Then the $n-D$ simplex can be written in the form

$$
\boldsymbol{X}=\left[\begin{array}{ccccc}
0 & 0 & 0 & \cdots & 0 \\
h_{1} & 0 & 0 & \cdots & 0 \\
r_{1} & h_{2} & 0 & \cdots & 0 \\
r_{1} & r_{2} & h_{3} & \cdots & 0 \\
\cdots & \cdots & \cdots & \cdots & \cdots \\
r_{1} & r_{2} & r_{3} & \cdots & h_{n}
\end{array}\right]
$$

One can notice that $h_{j}$ (Equation (15)) is the height of $j-D$ symmetrical simplex and $r_{j}$ (Equation (16)) is the radius of $j-D$ sphere (for $j=2$ - circle, for $j=3$ - "normal" sphere) inscribed in $j-D$ simplex. Moreover, we have the relations:

$$
h_{j}=r_{j}+R_{j} \text { and } R_{j}=j \cdot r_{j}
$$

where $R_{j}$ is the radius of $j$-D sphere circumscribed on $j$-D simplex, see Figure 5 .

In particular, for $n=7$, the matrix $X$ (Equation (13)) has 7 columns and 8 rows.

$$
\boldsymbol{X}=\left[\begin{array}{ccccccc}
0 & 0 & 0 & 0 & 0 & 0 & 0 \\
1 & 0 & 0 & 0 & 0 & 0 & 0 \\
0.5 & 0.866 & 0 & 0 & 0 & 0 & 0 \\
0.5 & 0.289 & 0.817 & 0 & 0 & 0 & 0 \\
0.5 & 0.289 & 0.204 & 0.791 & 0 & 0 & 0 \\
0.5 & 0.289 & 0.204 & 0.158 & 0.775 & 0 & 0 \\
0.5 & 0.289 & 0.204 & 0.158 & 0.129 & 0.764 & 0 \\
0.5 & 0.289 & 0.204 & 0.158 & 0.129 & 0.109 & 0.756
\end{array}\right] A_{0}
$$

\section{Translation and Reflection of the Simplex}

A simplex can be translated and/or rotated in space. Particularly, the simplex defined by $\boldsymbol{X}$ (Equation (13)), with the centre at $A_{c}=\left(r_{1}, \cdots, r_{n}\right)$, can be parallely shifted to the centre $P_{c 0}=(0, \cdots, 0)$ of the co-ordinate system. This way, one obtains a new matrix $\boldsymbol{X}_{t r}$

$$
\boldsymbol{X}_{t r}=\left[\begin{array}{cccccc}
-r_{1} & -r_{2} & -r_{3} & \cdots & -r_{n-1} & -r_{n} \\
R_{1} & -r_{2} & -r_{3} & \cdots & -r_{n-1} & -r_{n} \\
0 & R_{2} & -r_{3} & \cdots & -r_{n-1} & -r_{n} \\
\cdots & \cdots & \cdots & \cdots & \cdots & \cdots \\
0 & 0 & 0 & \cdots & R_{n-1} & -r_{n} \\
0 & 0 & 0 & \cdots & 0 & R_{n}
\end{array}\right]
$$




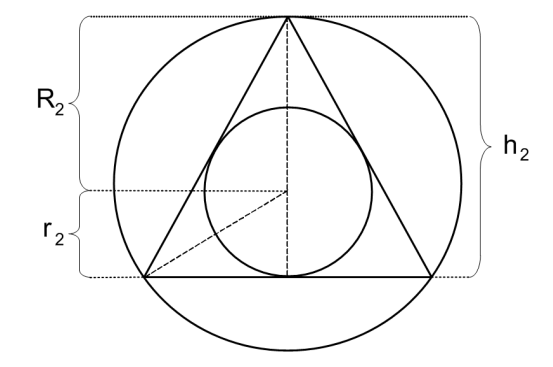

Figure 5. Graphical presentation of $r_{2}, h_{2}$ and $R_{2}$ in 2-D space (Equations (11) and

(12)) for $n=2$.

with $R_{j}$ and $r_{j}(j=1, \cdots, n)$ as ones in Equations (11), (12) and (14). Reflection of particular vertices of the matrix $\boldsymbol{X}_{t r}$ in the centre of co-ordinate system $(0, \cdots, 0)$ gives the simplex described by the matrix

$$
\boldsymbol{X}^{*}=\boldsymbol{X}_{t r} \cdot(-\boldsymbol{I})=\left[\begin{array}{cccccc}
r_{1} & r_{2} & r_{3} & \cdots & r_{n-1} & r_{n} \\
-R_{1} & r_{2} & r_{3} & \cdots & r_{n-1} & r_{n} \\
0 & -R_{2} & r_{3} & \cdots & r_{n-1} & r_{n} \\
\cdots & \cdots & \cdots & \cdots & \cdots & \cdots \\
0 & 0 & 0 & \cdots & -R_{n-1} & r_{n} \\
0 & 0 & 0 & \cdots & 0 & -R_{n}
\end{array}\right]
$$

where $\boldsymbol{I}=\left[\delta_{i j}\right]$-identity matrix.

The positions of the simplex presented in Figure 6 (in 2-D space), obtained from (I) by translation (II) and mirror reflection (III) in the origin of ordinate axis, are presented by matrices: $\boldsymbol{X}_{t r}$ and: $\boldsymbol{X}^{*}$ (Equation (21) for $n=2$ and

$$
\begin{gathered}
\boldsymbol{X}_{t r}=\left[\begin{array}{cc}
-r_{1} & -r_{2} \\
R_{1} & -r_{2} \\
0 & R_{2}
\end{array}\right]=\left[\begin{array}{cc}
-0.5 & -0.289 \\
0.5 & -0.289 \\
0 & 0.578
\end{array}\right] \\
\boldsymbol{X}^{*}=\left[\begin{array}{cc}
r_{1} & r_{2} \\
-R_{1} & r_{2} \\
0 & -R_{2}
\end{array}\right]=\left[\begin{array}{cc}
0.5 & 0.289 \\
-0.5 & 0.289 \\
0 & -0.578
\end{array}\right]
\end{gathered}
$$

Note that the number of $k-D$ walls in $n-D$ simplex equals to the number of $k+1$ point subsets $(k-D$ walls) generated from the $n+1$-point set. From the elementary theory of combinations it results that the number of $k-D$ walls equals to the number of possible combinations of $n+1$ items taken $k+1$ at a time, i.e.

$$
N(n, k)=\left(\begin{array}{l}
n+1 \\
k+1
\end{array}\right)=\frac{(n+1) !}{(k+1) !(n-k) !}
$$

Particularly, 3-D simplex (tetrahedron, $n=3$ ) involves $N(3,2)=4$ triangles ( $2-D$ walls), $N(3,1)=6$ edges (segments, $1-D$ walls) and $N(3,0)=4$ points (vertices, $0-D$ walls). Generally, the $n-D$ simplex consists of: $N(n, 0)=n+1$ vertices of the virtual solid stretched on them, $N(n, 1)=n(n+1) / 2$ edges, $\cdots$, $N(n, n-1)=n+1$ of $(\mathrm{n}-1)-D$ walls opposite to particular vertices.

\section{Simplex Optimization Procedure (SOP)}

The simplex method, based on the simplex concept, is considered among the most efficient optimization procedures, successfully applied in different areas of optimization techniques and chemical analyses.The simplex concept is applicable, among others, for optimisation of analytical methods [13], e.g. for searching the conditions 
(I)

(II)

(III)

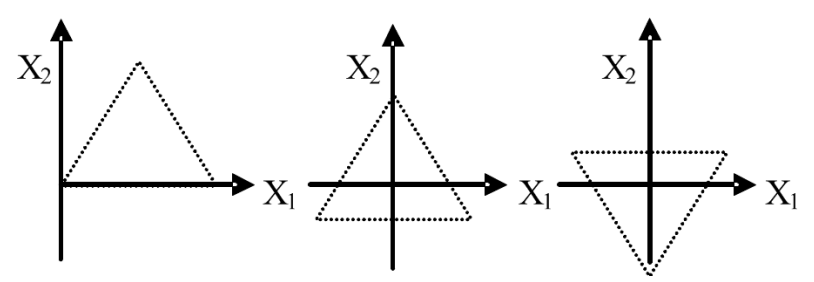

Figure 6. The simplexes obtained after successive (II) translation and (III) reflection of the original simplex (I) in the center of co-ordinate axes in 2-D space; for details-see text.

securing optimal accuracy and precision of an analytical method.

\subsection{An Objective Function}

The simplex concept is applicable, among others, for optimisation of analytical methods [13]. The variables, $x_{i}$ (scalars), named as factors forming the vector $\boldsymbol{x}$, affect the variable $y$, named as objective ("target") function, i.e.,

$$
y=y(\boldsymbol{x})=y\left(x_{1}, \cdots, x_{n}\right)
$$

The essence of optimisation is inherent in the objective function. Accuracy and precision are among the most important criteria of analytical methods. Another object functions are related to sensitivity of a method, efficiency of a chemical reaction, etc.

Let us assume that an analytical method aims to find the conditions securing true contents $\left(m_{0}, g / g\right)$ of an analyte in a sample to be obtained. For this purpose, the following criterion (objective function)

$$
y(i)=\frac{1}{N_{i}} \sum_{k=1}^{N_{i}}\left(m_{i k}-m_{0}\right)^{2}=\left(\bar{m}_{i}-m_{0}\right)^{2}+\left(1-\frac{1}{N_{i}}\right) \cdot s_{i}^{2}
$$

has been suggested [19], where:

$$
\begin{gathered}
\bar{m}_{i}=\frac{1}{N_{i}} \cdot \sum_{k=1}^{N_{i}} m_{i k} \\
s_{i}^{2}=\frac{1}{N_{i}-1} \cdot \sum_{k=1}^{N_{i}}\left(m_{i k}-\bar{m}_{i}\right)^{2}
\end{gathered}
$$

were calculated in the $i$-th simplex point, $A_{i}$ (Equation (3)), on the basis of $N_{i}$ measurements $m_{i k}$ of an intensive variable $m$ (e.g. number of grams of an analyte contained in a unit mass of solution); $m_{0}$ is the true value of this variable, obtained according to a reference method. In the object function (20), both terms, i.e. accuracy $\left(\bar{m}-\mathrm{m}_{0}\right)$ and precision, expressed by standard deviation, $s_{i}=\sqrt{s_{i}^{2}}$, are considered nearly equivalently; the difference in "weights": 1 and $1-1 / N_{i}$, of the two characteristics of the method becomes less significant at higher $N_{i}$ values.

The function (20) is an example of superposition of different characteristics of a method, i.e. accuracy and precision. The optimisation is terminated when the systematic error is insignificant, i.e., the inequality

$$
\left|\bar{m}_{i}-m_{0}\right|<\frac{s_{i}}{\sqrt{N_{i}}} \cdot t\left(0.95, f_{i}\right)
$$

is fulfilled; $t\left(0.95, f_{i}\right)$ is the critical $t$-value of the Student's $t$-test at $f_{i}=N_{i}-1$ degrees of freedom, on $95 \%$ probability level pre-assumed. In particular, for $N_{i}=4$, i.e. $f_{i}=3$, we have $t(0.95,3)=3.182$, and then Equation (23) has the form

$$
\left|\bar{m}_{i}-m_{0}\right|<1.591 \cdot s_{i}
$$




\subsection{Decision Variables and Arrangement of Experimental Conditions}

The optimization procedure assumes:

1) Selection of natural decision variables, $Z_{i}$, as independent (in principle) individual factors affecting the values of the objective function (Equation (19)) and

2) Their order, $Z_{j}(j=1, \cdots, n)$.

The number $(n)$ of the variables $Z_{j}$ chosen defines the simplex dimension.

The kind $\left(Z_{j}\right)$ and the number $(n)$ of these variables that assume continuous (not discrete) values $z_{j}(j=1, \cdots, n)$, consisting the vector $z=\left(z_{1}, \cdots, z_{n}\right)^{\mathrm{T}}$, affects the success (efficiency) of optimisation process aiming to get optimal (maximal or minimal) value for the object function, e.g. minimal value for $y=y(i)$ defined by Equation (20). It should be noted that the $n-D$ simplex matrix (Equation (5)) refers-in principle- to orthogonal (independent) variables. The independent variables assuming continuous values are, among others: $\mathrm{pH}$, temperature, time, rate of an operation.

One should be noticed that the form of the matrix (5) enables to add a new variable, $Z_{n+1}$, to the set of $n$ variables $Z_{1}, \cdots, Z_{n}$ assumed at the start for optimization-provided that the decision of inclusion of variable $Z_{n+1}$ into the set of variables has been undertaken after measurements done in the points $A_{0}, \cdots, A_{p}$, at $p \leq n$, where $Z_{n+1}$ remained unchanged. In this case, the measurements done at the points $A_{0}, \cdots, A_{p}$ has not to be repeated.

\subsection{Design of Experiments and Optimization within the Starting Simplex}

The introductory step of the optimization procedure assumes fixing the starting values, $z_{0 j}$, and steps, $\Delta z_{j}$, assumed for $Z_{j}(j=1, \cdots, n)$; these values affect strongly the efficiency of the optimization procedure. Some constraints put on $z_{j}$ and $\Delta z_{j}$ values (resulting from physicochemical, chemical or technological reasons) can also be taken into account. These constraints may result e.g., from limited solubility, possibility of phase change affected by temperature, etc.

The optimization is realized there for values of natural variables calculated from the formula

$$
z_{i j}=z_{0 j}+x_{i j} \cdot \Delta z_{j}
$$

where $i=0,1, \cdots, n$ enumerates successive experimental points realized within the initial simplex. The advised values for $x_{i j}$ in (5) are defined in the matrix (13) and specified e.g. in the matrix (15) if $n=7$. The values $y(j), j=0, \cdots, n$, obtained at $n+1$ points $A_{0}, A_{1}, \cdots, A_{n}$ of the initial simplex are the basis for its further evolution of the simplex.

\subsection{Optimization at the Points of Evolving Simplex}

At the first stage of the simplex evolution (Figure 7), a point $A_{w 1}$ with the worst $(w 1)$ value, $y(w 1)$, for the object function (Equation (20)) is indicated, $w 1 \in<0, n>$. The point $A_{w 1}$ is replaced by a new point, $A_{n+1}$, obtained by reflection of $A_{w 1}$ in the centre, $A_{c 1}\left(x_{c 1}\right)$, of the remaining $n$ points of the initial simplex, i.e., in $n-1-D$ wall not containing the point $A_{w 1}$. In other words, $\boldsymbol{x}_{\mathrm{c} 1}$ is the middle point between $\boldsymbol{x}_{\mathrm{w} 1}$ and $x_{\mathrm{n}+1}$, i.e.,

$$
\frac{\boldsymbol{x}_{w 1}+\boldsymbol{x}_{n+1}}{2}=\boldsymbol{x}_{c 1}=\frac{1}{n} \cdot \sum_{i \neq w 1} \boldsymbol{x}_{i}
$$

After adding the term $(1 / n) \cdot x_{w 1}$ to both sides of Equation (26), we obtain the co-ordinates of the new point, $A_{n+1}$,

$$
x_{n+1}=\frac{2}{n} \cdot \sum_{i=1}^{n} x_{i}-\left(1+\frac{2}{n}\right) \cdot x_{w 1}
$$

After measurement(s) done at the point $A_{n+1}$, the values $y(i)$ are compared again within the new (evolved) simplex (containing $A_{n+1}$ and not containing $A_{w 1}$ ) and the point $A_{w 2}$ with the worst value, $y(w 2)$ is chosen. Then $A_{w 2}$ is reflected in the centre of $n-1-D$ wall of the new simplex (not containing w2 ). This way, a new point $A_{n+2}$ is obtained and further operations are repeated again, in cyclic manner. At $q$-th reflection one can write 


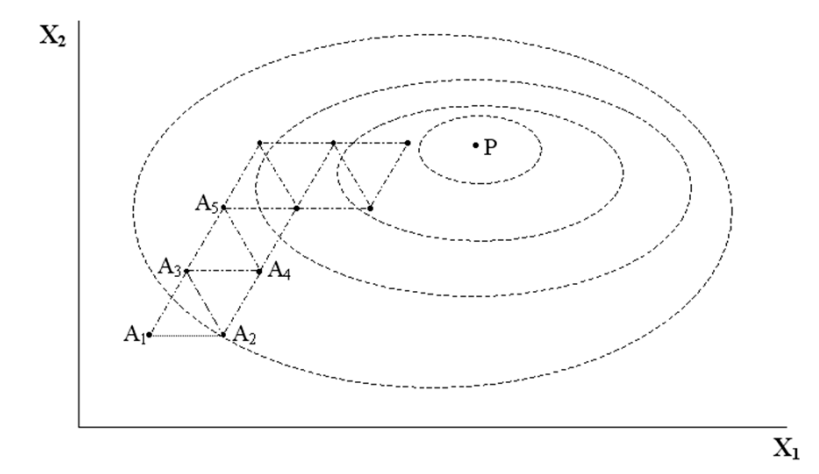

Figure 7. Evolving simplex in 2-D space.

$$
\frac{\boldsymbol{x}_{w q}+\boldsymbol{x}_{n+q}}{2}=\boldsymbol{x}_{c q}=\frac{1}{n} \cdot \sum_{i \neq w q}^{(q)} \boldsymbol{x}_{i}
$$

In the example presented above, the initial (symmetrical) shape of the evolving simplex is maintained owing to the fact that 1) mirror reflections are applied and 2) equal statistical weights are assumed to all the simplex points considered. Sometimes we are forced to move the simplex within a constrained component space [20].

The evolving simplex approaches the quasi-optimal region, where $y(i)$ values do not change distinctly. In this region, the evolving simplex should be contracted or diminished (Figure 8); such operations enter e.g. the MINUIT program. The contraction/dilatation and weighting are involved in the relation [21] [22]

$$
\boldsymbol{x}_{n+q}=(1-\alpha) \cdot \boldsymbol{x}_{w q}+\alpha \cdot \boldsymbol{x}_{c q}
$$

where the parameter $\alpha$ results from re-parametrization of the a line passing through 2 points: one of them is $\boldsymbol{x}_{w q}$, the second one is the centre $\boldsymbol{x}_{c q}$ of area of $q$-th, $\mathrm{n}-1-D$ simplex, defined as follows

$$
\boldsymbol{x}_{c q}=\frac{\sum_{i \neq w q}^{(q)} p_{i} \boldsymbol{x}_{i}}{\sum_{i \neq w q}^{(q)} p_{i}}
$$

where the operator $\sum_{i \neq w q}{ }^{(q)}$ means summation within $q$-th $\mathrm{n}-1-D$ simplex ( $\boldsymbol{x}_{w q}$ excluded). The contraction or expansion and weighting (“weights” $p_{i}$ ) different points $\boldsymbol{x}_{i}$ deforms the evolving simplex gradually. As a rule, it is not an advantageous occurrence during the optimization procedure, however. The simplex deformation is influenced mainly by the weighting factors [23]. Moreover, a shape of response function may sometimes cause the optimum searching impossible, even in $2-D$ space.

\section{Example of SOP}

To illustrate the principle of SOP, a simple example of gravimetric analysis is considered below. The matrix expressed by Equation (15) was chosen for initial simplex, and Equation (20) was applied as criterion of optimization. $N_{i}=4$ measurements were made at each simplex point. In our case, the relation (24) is chosen as the criterion of optimization. Analyses were made with $\mathrm{CdSO}_{4}$ solution of $\left(m_{0}=0.01272 \mathrm{gCd} / 1 \mathrm{~g}\right)$, standardised according to electrogravimetry, considered as the reference method.

\subsection{Analytical Prescription}

The samples, ca. $5 \mathrm{~mL}$ of the solution, weighed on analytical balance $( \pm 0.1 \mathrm{mg})$, were taken for analysis. The samples were treated with tartaric acid $(0.220 \mathrm{~g} / \mathrm{mL})$ solution and then with $2.51 \mathrm{~mol} / \mathrm{L} \mathrm{NaOH}$ solution. After dilution with water, the solution was treated with 8-hydroxyquinoline (HL) solution $(3.00 \% \mathrm{~m} / \mathrm{v}$, in ethanol) added dropwise from burette, with defined rate of addition. The mixture was leaved for defined time on the water-bath for re-crystallisation of the precipitate $\mathrm{CdL}_{2}$ at defined temperature, then filtered, washed with water, dried to a constant mass at $105^{\circ} \mathrm{C}$ and weighed. 


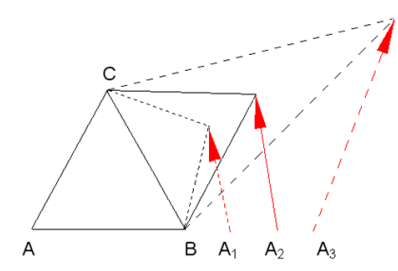

(a)

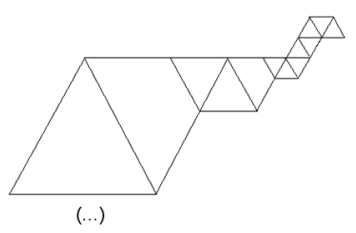

(b)

Figure 8. (a) The contraction $\left(A_{1}\right)$, reflection $\left(A_{2}\right)$ i expansion $\left(A_{3}\right)$ of the vertex $A$ of the simplex $\mathrm{ABC}(n=2)$ on the edge $\mathrm{BC}$; and (b) Diminution of the simplex.

\subsection{Decision Variables}

$\mathrm{Z}_{1}$-re-crystallisation time [min] of the precipitated $\mathrm{CdL}_{2}$;

$\mathrm{Z}_{2}$-mass [g] of tartaric acid added in the solution;

$\mathrm{Z}_{3}$-volume $[\mathrm{ml}]$ of $\mathrm{NaOH}$ added;

$\mathrm{Z}_{4}$-volume [ml] of 8-hydroxyquinoline added;

$\mathrm{Z}_{5}$-rate $[\mathrm{ml} / \mathrm{min}]$ of 8-hydroxyquinoline addition;

$\mathrm{Z}_{6}$ - temperature $\left[{ }^{\circ} \mathrm{C}\right]$ of water bath where re-crystallization of $\mathrm{CdL}_{2}$ occurs;

$\mathrm{Z}_{7}$-volume [ml] of the solution before precipitation.

For example, the value $z_{04}=7.20[\mathrm{ml}]$ refers to variable $Z_{4}$; the related value $\Delta z_{4}=1.00 \mathrm{ml}$. For $i=3$ and $j=4$, from Table 1 we have $z_{34}=8.00+0.817 \times 1.00 \cong 8.82$.

\subsection{Results of Analyses within the Initial Simplex}

The simplex optimisation procedure was realized in experiments performed in all points of the initial simplex (rows $0, \cdots, 7$ in Table 1 ). In each point $A_{i}(i=0, \cdots, 7), N_{i}=4$ measurements were done. The results $\mathrm{m}_{\mathrm{ik}}[\mathrm{gCD} / \mathrm{g}]$ are presented in Table 2.

On the basis of the data in Table 2, the $y(i)$ values were calculated (last column in Table 1).

As results from detailed calculations done on the basis of results in Table 2, the inequality (23) has not been fulfilled within the initial simplex and then the evolution of the simplex was needed.

Referring to the Table 1, the worst $(w=w 1)$ result within the initial simplex was obtained at the point $A_{2}$. During the simplex evolution, this point was reflected in the centre $\boldsymbol{x}_{\mathrm{c} 1}$ of the remaining points of the starting simplex. Referring to Table 1, we have, for example,

$$
\begin{gathered}
x_{2 c 1}=(2 \cdot 0+5 \times 0.289) / 7=0.206 \\
\left(0.866+x_{82}\right) / 2=0.206 \rightarrow x_{82}=-0.454 \\
z_{82}=3.00+(-0.454) \times 1 \cong 2.55 \\
x_{3 c 1}=(2 \times 0+0.817+4 \times 0.204) / 7=0.233 \\
\left(0+x_{83}\right) / 2=0.233 \rightarrow x_{83}=0.466 \\
z_{83}=8.00+0.466 \times 1 \cong 8.47
\end{gathered}
$$

At the point $A_{8}$, the condition (24) has not been fulfilled as well, and further evolution of the simplex was required. The worst $y(w 2)$ value within the new simplex was stated in the point $A_{7}$. Further calculations referred to the new point $A_{9}$ are exemplified below:

$$
\begin{gathered}
x_{2 c 2}=(2 \times 0+4 \times 0.289+1 \times(-0.454)) / 7=0.1004 \\
\left(0.289+x_{92}\right) / 2=0.1004 \rightarrow x_{92}=-0.088, \\
z_{92}=3.00+(-0.088) \times 1 \cong 2.91 ;
\end{gathered}
$$


Table 1. The values for $\mathrm{z}_{\mathrm{ij}}$ assumed in points of the initial (columns 1-7, rows 0-7) and evolving (columns 1-7, rows 8-10) simplexes. For all points, the values $y(i)$ were obtained on the basis of $N_{i}=4$ measurements.

\begin{tabular}{|c|c|c|c|c|c|c|c|c|c|}
\hline \multicolumn{2}{|c|}{$j \triangleright$} & 1 & 2 & 3 & 4 & 5 & 6 & 7 & \multirow{2}{*}{$y(i) \cdot 10^{8}$} \\
\hline$i \nabla$ & $\Delta z_{i}$ & 60 & 1.00 & 1.00 & 1.00 & 1.00 & 10 & 50 & \\
\hline 0 & $z_{0 j}$ & 60 & 3.00 & 8.00 & 7.20 & 1.00 & 60 & 100 & 1.2 \\
\hline 1 & $z_{1 j}$ & 120 & 3.00 & 8.00 & 7.20 & 1.00 & 60 & 100 & 0.7 \\
\hline 2 & $z_{2 j}$ & 90 & 3.87 & 8.00 & 7.20 & 1.00 & 60 & 100 & 3.0 \\
\hline 3 & $z_{3 j}$ & 90 & 3.29 & 8.82 & 7.20 & 1.00 & 60 & 100 & 1.9 \\
\hline 4 & $Z_{4 j}$ & 90 & 3.29 & 8.20 & 7.99 & 1.00 & 60 & 100 & 0.7 \\
\hline 5 & $z_{5 j}$ & 90 & 3.29 & 8.20 & 7.36 & 1.78 & 60 & 100 & 1.3 \\
\hline 6 & $z_{6 j}$ & 90 & 3.29 & 8.20 & 7.36 & 1.13 & 67.5 & 100 & 0.8 \\
\hline 7 & $z_{7 j}$ & 90 & 3.29 & 8.20 & 7.36 & 1.13 & 61 & 138 & 2.3 \\
\hline 8 & $z_{8 j}$ & 90 & 2.55 & 8.47 & 7.56 & 1.30 & 62.5 & 111 & 1.3 \\
\hline 9 & $Z_{9 j}$ & 90 & 2.91 & 8.34 & 7.46 & 1.21 & 62 & 65 & 1.8 \\
\hline 10 & $z_{10, j}$ & 90 & 2.80 & 7.59 & 7.69 & 1.40 & 63 & 93 & 0.5 \\
\hline
\end{tabular}

Table 2. Results of measurements done within the initial simplex.

\begin{tabular}{cccccrrrr}
\hline $\mathrm{A}_{0}$ & $\mathrm{~A}_{1}$ & $\mathrm{~A}_{2}$ & $\mathrm{~A}_{3}$ & $\mathrm{~A}_{4}$ & $\mathrm{~A}_{5}$ & $\mathrm{~A}_{6}$ & 0.01262 & 0.01255 \\
0.01260 & 0.01265 & 0.01256 & 0.01256 & 0.01265 & 0.01262 & 0.01268 \\
0.01263 & 0.01268 & 0.01252 & 0.01258 & 0.01267 & 0.01255 & 0.01259 \\
0.01257 & 0.01259 & 0.01249 & 0.01269 & 0.01260 & 0.01267 & 0.01261 & 0.01262 \\
0.01268 & 0.01265 & 0.01267 & 0.01255 & 0.01264 & 0.01262 & 0.01264 & 0.01253 \\
\hline
\end{tabular}

$$
\begin{gathered}
x_{3 c 2}=(2 \times 0+0.817+3 \times 0.204+0.467) / 7=0.271 \\
\left(0.204+x_{93}\right) / 2=0.271 \rightarrow x_{93}=0.338 \\
z_{93}=8.00+0.338 \times 1 \cong 8.34 \\
x_{7 c 2}=(6 \times 0+0.216) / 7=0.031 \\
\left(0.756+x_{97}\right) / 2=0.031 \rightarrow x_{97}=-0.694, \\
z_{97}=100+(-0.694) \times 50 \cong 65 .
\end{gathered}
$$

The coordinates of the new points: $A_{8}, A_{9}$ and $A_{10}$ for evolving simplexes, calculated with use of the coded variables $x_{i j}$ specified.

$$
\left[\begin{array}{ccccccc}
0.5 & -0.454 & 0.467 & 0.361 & 0.295 & 0.249 & 0.216 \\
0.5 & -0.088 & 0.338 & 0.261 & 0.214 & 0.180 & -0.694 \\
0.5 & -0.196 & -0.412 & 0.494 & 0.404 & 0.341 & -0.137
\end{array}\right] \begin{aligned}
& A_{8} \\
& A_{9} \\
& A_{10}
\end{aligned}
$$

The optimisation procedure has been terminated at the point $A_{10}$, where $\bar{m}_{i}=0.01267$ and variance $s_{i}{ }^{2}=0.32 \times 10^{-8}$ were found on the basis of 4 measurements and then the inequality (24)

$$
|0.01267-0.01272|<0.56 \times 10^{-4} \times 1.591=0.89 \times 10^{-4}
$$

was fulfilled and

$$
y(10)=(0.01267-0.01272)^{2}+(1-1 / 4) \times 0.32 \times 10^{-8}=0.49 \times 10^{-8} \cong 0.5 \times 10^{-8}
$$

\subsection{Optimal Prescription}

On the basis of the optimization procedure, one can formulate the following prescription.

The samples, ca. $5 \mathrm{ml}$ of the standardised $\mathrm{CdSO}_{4}$ solution, weighed on analytical balance $( \pm 0.1 \mathrm{mg})$, were 
taken for analysis. The solution was treated with $2.80 \mathrm{~g}$ of tartaric acid and then with $7.9 \mathrm{ml}$ of $2.5 \mathrm{~mol} / \mathrm{l} \mathrm{NaOH}$ solution. After dilution with water up to ca $90 \mathrm{ml}$, the solution was treated with $7.7 \mathrm{ml}$ of $3 \%(\mathrm{~m} / \mathrm{v})$ 8-hydroxyquinoline solution (added dropwise from burette, with the rate $1.4 \mathrm{ml} / \mathrm{min}$. The mixture was leaved for ca. 90 min on the water-bath for re-crystallisation of the precipitate $\mathrm{CdL}_{2}$ at temperature $63^{\circ} \mathrm{C}$, then filtered, washed with water, dried to a constant mass at $105^{\circ} \mathrm{C}$ and weighed.

\section{Final Comments}

In this paper, the simplex method has been presented in terms known from elementary algebra and geometry. On the basis of scalar product and length of vectors in normalized scale, the co-ordinates of simplex vertices in $n-D$ space were determined. A criterion of optimization, where accuracy and precision of measurements were considered equivalently, has been suggested.

The simplex method was considered as the most efficient among optimization procedures, applicable in simulating procedures realized in recursive computer programs used for simulation purposes and in optimization of analytical procedures. The simplex method requires only one additional experiment, regardless of the number of factors being varied. This drastically reduces the number of experiments required to reach the optimum.

\section{References}

[1] Anderson, V.L. and McLean, R.A. (1974) Design of Experiments: A Realistic Approach. Marcel Dekker, Inc., New York, 363.

[2] Nelder, J.A. and Mead, R. (1965) A Simplex Method for Function Minimization. Computer Journal, 7, 308-313. http://dx.doi.org/10.1093/comjnl/7.4.308

[3] Walters, F.H., Parker, L.R., Morgan, S.L. and Deming, S.N. (1991) Sequential Simplex Optimization. CRC Press, Boca Raton. http://www.chem.sc.edu/faculty/morgan/pubs/SequentialSimplexOptimization.pdf

[4] Spendley, W., Hext, G.R. and Himsworth, F.R. (1962) Sequential Application of Simplex Designs in Optimisation and Evolutionary Operation. Technometrics, 4, 441-461. http://dx.doi.org/10.1080/00401706.1962.10490033

[5] Fletcher, R. (1965) Function Minimization without Evaluating Derivatives-A Review. Computer Journal, 8, 33-41. http://dx.doi.org/10.1093/comjnl/8.1.33 http://folk.uib.no/ssu029/Pdf_file/Fletcher65.pdf

[6] Olsson, D.M. and Nelson, L.S. (1975) The Nelder-Mead Simplex Procedure for Function Minimization. Technometrics, 17, 45-51. http://dx.doi.org/10.1080/00401706.1975.10489269

[7] Fletcher, R. and Powell, M.J.D. (1963) A Rapidly Convergent Descent Method for Minimization. Computer Journal, 6, 163-168. http://dx.doi.org/10.1093/comjnl/6.2.163

[8] Fletcher, R. and Reeves, C.M. (1964) Function Minimization by Conjugate Gradients. Computer Journal, 7, $149-154$. http://dx.doi.org/10.1093/comjnl/7.2.149

[9] Lagarias, J.C., Reeds, J.A., Wright, M.H. and Wright, P.E. (1998) Convergence Properties of the Nelder-Mead Simplex Algorithm in Low Dimensions. SIAM Journal of Optimization, 9, 112-147. http://dx.doi.org/10.1137/S1052623496303470 http://citeseerx.ist.psu.edu/viewdoc/download?doi=10.1.1.120.6062\&rep=rep1\&type=pdf

[10] Box, G.E.P. and Hunter, J.S. (1957) Multi-Factor Experimental Designs for Exploring Response Surfaces. Annals of Mathematical Statistics, 28, 195-241. http://dx.doi.org/10.1214/aoms/1177707047

[11] Box, G.E.P. and Draper, N.R. (1969) Evolutionary Operation. John Wiley \& Sons, Inc., New York.

[12] Massart, D.L., Vanderginste, B.G.M., Deming, S.N., Michotte, Y. and Kaufman, L. (1988) Chemometrics: A Textbook. Elsevier, Amsterdam.

[13] Massart, D.L., Vanderginste, B.G.M., Buydens, L.M.C., De Jong, S., Lewi, P.J. and Smeyers-Verbeke, J. (1997) Handbook of Chemometrics and Qualimetrics. In: Data Handling in Science and Technology, Vol. 22, Elsevier, Amsterdam.

[14] Box, G.E.P. (1957) Evolutionary Operation: A Method for Increasing Industrial Productivity. Journal of the Royal Statistical Society. Series C (Applied Statistics), 6, 81-101. http://en.wikipedia.org/wiki/EVOP

[15] Hahn, G.J. (1976) Process Improvement Using Evolutionary Operation. 204-206. http://rube.asq.org/statistics/2011/11/quality-tools/process-improvement-through-simplex-evop.pdf

[16] James, F. (2004) MINUIT Tutorial, Function Minimization, Geneva. Reprinted from the Proceedings of the 1972 CERN Computing and Data Processing School, Pertisau, 10-24 September 1972 (CERN 72-21). 
http://seal.web.cern.ch/seal/documents/minuit/mntutorial.pdf

[17] Liu, Q. (2001) Implementing Reusable Mathematical Procedures Using C++, C/C++. Users Journal.

[18] Walters, F.H., Parker Jr., L.R., Morgan, S.L. and Deming, S.N. (1991) Sequential Simplex Optimization. CRC Press, Boca Raton.

[19] Michałowski, T., Rokosz, A. and Wójcik, E. (1980) Optimization of the Conventional Method for Determination of Zinc as 8-Oxyquinolate in Alkaline Tartrate Medium. Chemia Analityczna, 25, 563-566.

[20] Palasota, J.A., Leonidou, I., Palasota, J.M., Chang, H.-L. and Deming, S.N. (1992) Sequential Simplex Optimization in a Constrained Simplex Mixture Space in Liquid Chromatography. Analytica Chimica Acta, 270, 101-106. http://dx.doi.org/10.1016/0003-2670(92)80096-P

[21] Deming, S.N. and Morgan, S.L. (1973) Simplex Optimization of Variables in Analytical Chemistry. Analytical Chemistry, 45, 278A-283A.

[22] Deming, S.N. and Morgan, S.L. (1983) Teaching the Fundamentals of Experimental Design. Analytica Chimica Acta, 150, 183-198. http://dx.doi.org/10.1016/S0003-2670(00)85470-7

[23] Umeda, T. and Ichikawa, A. (1971) A Modified Complex Method for Optimization. Industrial \& Engineering Chemistry Process Design and Development, 10, 229-236. 
Scientific Research Publishing (SCIRP) is one of the largest Open Access journal publishers. It is currently publishing more than 200 open access, online, peer-reviewed journals covering a wide range of academic disciplines. SCIRP serves the worldwide academic communities and contributes to the progress and application of science with its publication.

Other selected journals from SCIRP are listed as below. Submit your manuscript to us via either submit@scirp.org or Online Submission Portal.
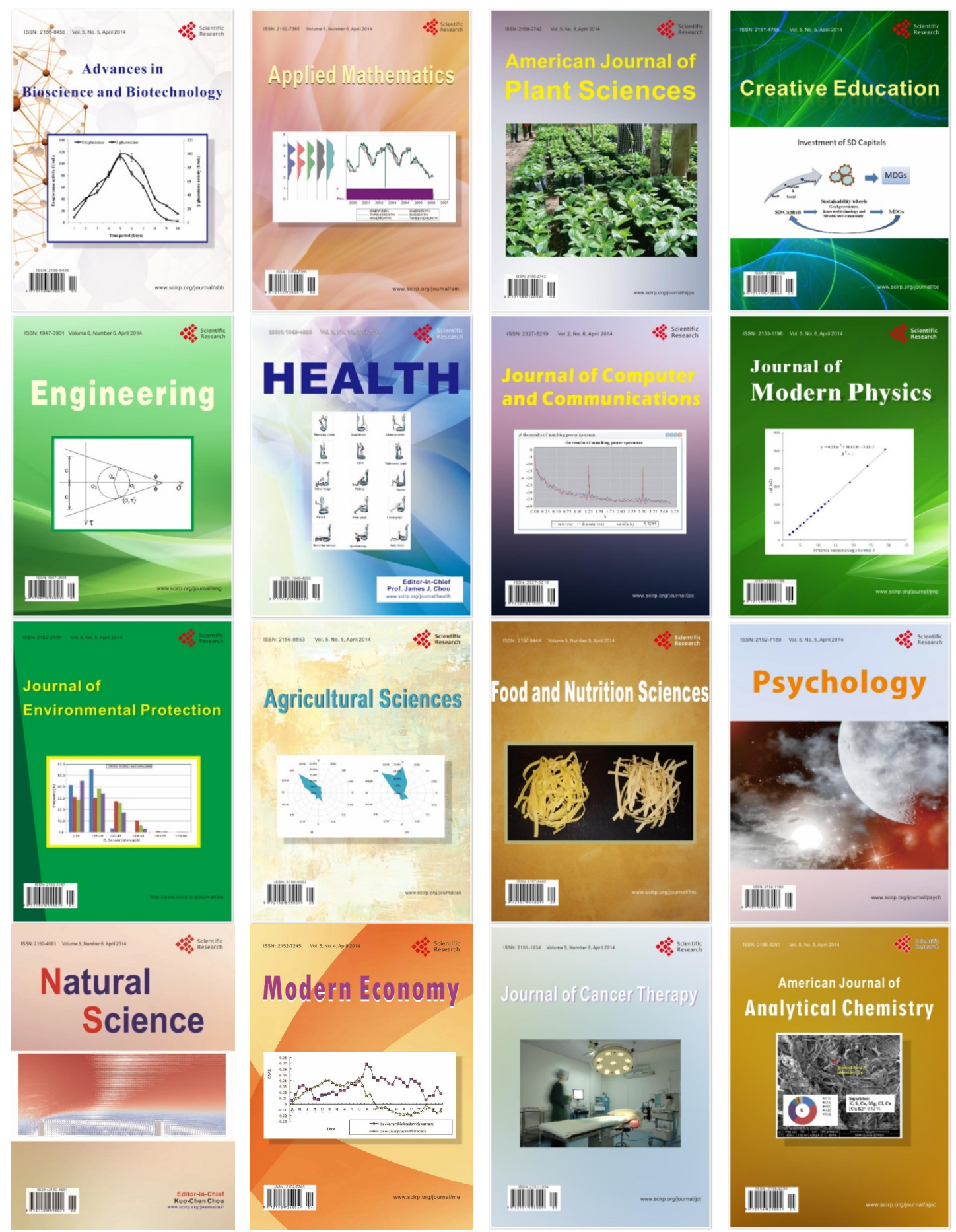